\title{
The Problem of Bicenter and Isochronicity for a Class of Quasi Symmetric Planar Systems
}

\author{
Du Chaoxiong \\ Department of Mathematics, Hunan Shaoyang University, Shaoyang, Hunan 422000, China \\ Correspondence should be addressed to Du Chaoxiong; ducx123@126.com
}

Received 19 February 2014; Revised 5 April 2014; Accepted 5 April 2014; Published 29 April 2014

Academic Editor: Tonghua Zhang

Copyright ( 2014 Du Chaoxiong. This is an open access article distributed under the Creative Commons Attribution License, which permits unrestricted use, distribution, and reproduction in any medium, provided the original work is properly cited.

\begin{abstract}
We study a class of quasi symmetric seventh degree systems and obtain the conditions that its two singular points can be two centers at the same step by careful computing and strict proof. In addition, the condition of an isochronous center is also given. In terms of quasi symmetric systems, our work is interesting and obtained conclusions about bicenters are new.
\end{abstract}

\section{Introduction}

One of the open problems for planar polynomial differential systems

$$
\frac{d x}{d t}=P(x, y), \quad \frac{d y}{d t}=Q(x, y)
$$

is how to characterize their centers and isochronous centers. Article [1] pointed out that "a center of an analytic system is isochronous if and only if there exists an analytic change of coordinates such that the original system is reduced to a linear system," so an isochronous center is also called a linearizable center. A center is an isochronous center or linearizable center if the period of all periodic solutions is constant.

The main method to investigate centers and isochronous centers problem is the computation of focus values and isochronous constants (see [2-13]), which is a kind of active effective method. The vanishing of all isochronous constants or period constants is a necessary and sufficient condition for the isochronicity. Although theoretically the isochronous center problem can be solved by using the method letting all period constants become zero, in fact only the first few period constants can be given in personal computer. Hence, up to now the sufficient and necessary condition determining an isochronous center can only be found by making some appropriate analytic changes of coordinates which let the original system be reduced to a linear system. This kind of appropriate analytic change is very difficult to be obtained, so only a handful of isochronous systems are investigated. Several classes of known studied isochronous systems are as follows: quadratic isochronous centers (see [14]); isochronous centers of a linear center perturbed by third, fourth, and fifth degree homogeneous polynomials (see $[3,4,15]$ ); complex polynomial systems (see [1]); reversible systems (see [12, 16]); and isochronous centers of quartic systems with degenerate infinity (see [17]).

For seventh degree system, [18] studied the limit cycles bifurcations. In this paper, we investigate the centers and isochronous centers problem for a class of seventh degree systems with the following form:

$$
\begin{gathered}
\frac{d x}{d t}=-(x \delta+y)\left(x^{2}+y^{2}\right)^{3}-P_{6}(x, y), \\
\frac{d y}{d t}=(x-y \delta)\left(x^{2}+y^{2}\right)^{3}+Q_{6}(x, y),
\end{gathered}
$$

in which

$$
\begin{aligned}
P_{6}(x, y)= & \left(1+a_{1}\right) x^{4} y+\left(3+2 a_{1}\right) x^{5} y+2 a_{4} x^{3} y^{2} \\
& +4 a_{4} x^{4} y^{2}-\left(a_{1}-a_{3}-2 a_{5}\right) x^{2} y^{3} \\
& +\left(3+2 a_{5}\right) \times x^{3} y^{3}+2 x\left(a_{6}+2 a_{4} x\right) y^{4}
\end{aligned}
$$




$$
\begin{aligned}
& -\left(a_{3}+2 a_{1} x-2 a_{5} x\right) y^{5}+\frac{1}{2} x^{2}(x-y) \\
& \times(x+y)\left(x+3 x^{2}+3 y^{2}\right) \delta \\
Q_{6}(x, y)= & \frac{1}{2} x^{5}(1+3 x)+a_{4} x^{4}(1+2 x) y \\
& -\frac{1}{2}\left(1+4 a_{1}-2 a_{5}\right) x^{3} y^{2}-\left(4 a_{1}-a_{5}\right) x^{4} y^{2} \\
& -\left(a_{4}-a_{6}\right) x^{2} y^{3}-\left(2 a_{3}+a_{5}\right) x y^{4} \\
& -\frac{1}{2}\left(3+8 a_{1}\right) x^{2} y^{4}-y^{5}\left(a_{6}+2 a_{4} x+a_{5} y\right) \\
& -x^{3} y\left(x+3 x^{2}+3 y^{2}\right) \delta
\end{aligned}
$$

and $\delta, a_{i}(i \in\{1,2,3,4,5\})$ are real numbers.

We obtain that the infinity and the elementary singular point $(-1 / 2,0)$ of $\left.(2)\right|_{\delta=0}$ have the same center condition and investigate the isochronous center condition of $(-1 / 2,0)$. What is worth pointing out is that the results of bicenters in a polynomial system of degree $n$ are less seen in published papers; our work is new and interesting.

In general, our investigations are shown as follows. Firstly, by making two appropriate transformations (i.e., (32) and (33)) of system (2), system (2) is transformed into system (34); hence, the problem of system (2) center problem and isochronicity is reduced to investigate system (34) center and the isochronous centers problem. Secondly, we prove that system $(34)$ is symmetric about $(-1,0)$. System $(34)$ has two symmetric elementary singular points (i.e., the origin and $(-2,0))$, which are from the infinity and the elementary focus $(-1 / 2,0)$ of $(2)$ under transformations (32) and (33). Thirdly, through calculating system (34) focal values when $\delta=0$ and careful analysis, we obtain the condition that the infinity and the elementary focus $(-1 / 2,0)$ of $\left.(2)\right|_{\delta=0}$ become bicenters at the same time. Lastly, we study the above isochronicity problems of $(-1 / 2,0)$. During the course of investigating isochronicity of system $\left.(2)\right|_{\delta=0}$, at first we make use of the method in [19] to compute the first several period constants and find the isochronous centers' necessary condition; next we try to find the sufficient condition. We obtain all sufficient and necessary conditions that the elementary focus $(-1 / 2,0)$ of (2) become an isochronous center.

The paper is organized as follows. In Section 2, we introduce preliminary methods to calculate focal values (or Lyapunov constants) and period constants which are necessary for our study in Sections 3 and 4. In Section 3, we make two appropriate transformations which let research on system (2) be reduced to investigate a class of symmetric seventh degree systems in which the first five focal values with more simple expressions are given. Being based on it, we find the condition that the infinity and the elementary singular points of (2) $\left.\right|_{\delta=0}$ can be bicenters and prove them. In Section 4, by analyzing all center conditions and all obtained expressions of periodic constants, we give all sufficient and necessary conditions that the elementary singular point $(-1 / 2,0)$ of $\left.(2)\right|_{\delta=0}$ becomes an isochronous center and prove them strictly.

\section{Preliminary Method to Compute Focal Values and Periodic Constant}

In order to continue this study, at first we introduce previous methods to calculate focal values and periodic constants which are necessary for us to verify centers and isochronous centers.

Consider the following real system:

$$
\begin{aligned}
& \frac{d x}{d t}=\delta x-y+\sum_{k=2}^{\infty} X_{k}(x, y), \\
& \frac{d y}{d t}=x+\delta y+\sum_{k=2}^{\infty} Y_{k}(x, y),
\end{aligned}
$$

where $X_{k}(x, y)$ and $Y_{k}(x, y)$ are homogeneous polynomials of degree $k$ about $x$ and $y$.

By means of transformation

$$
z=x+i y, \quad w=x-i y, \quad T=i t, \quad i=\sqrt{-1},
$$

system (4) $\left.\right|_{\delta=0}$ can be transformed into the following complex system:

$$
\begin{gathered}
\frac{d z}{d T}=z+\sum_{k=2}^{\infty} Z_{k}(z, w)=Z(z, w), \\
\frac{d w}{d T}=-w-\sum_{k=2}^{\infty} W_{k}(z, w)=-W(z, w),
\end{gathered}
$$

where $z, w, T$ are complex variables and

$$
Z_{k}(z, w)=\sum_{\alpha+\beta=k} a_{\alpha \beta} z^{\alpha} w^{\beta}, \quad W_{k}(z, w)=\sum_{\alpha+\beta=k} b_{\alpha \beta} w^{\alpha} z^{\beta} .
$$

Obviously, the coefficients of (6) satisfy conjugate condition; that is,

$$
\overline{a_{\alpha \beta}}=b_{\alpha \beta}, \quad \alpha \geq 0, \beta \geq 0, \alpha+\beta \geq 2
$$

System (4)| $\left.\right|_{\delta=0}$ and system (6) are called concomitant systems (for the definition see $[11,12]$ ).

For the complex analytic system (6), making transformation

$$
z=r e^{i \theta}, \quad w=r e^{-i \theta}, \quad T=i t
$$

system (6) can be transformed into

$$
\begin{aligned}
\frac{d r}{d t} & =i \frac{w Z-z W}{2 r}=i r \sum_{k=1}^{\infty} \frac{w Z_{k+1}-z W_{k+1}}{2 z w} \\
& =\frac{i r}{2} \sum_{m=1}^{\infty} \sum_{\alpha+\beta=m+2}\left(a_{\alpha, \beta-1}-b_{\beta, \alpha-1}\right) e^{i(\alpha-\beta) \theta} r^{m},
\end{aligned}
$$




$$
\begin{aligned}
\frac{d \theta}{d t} & =\frac{w Z+z W}{2 z w}=1+\sum_{k=1}^{\infty} \frac{w Z_{k+1}+z W_{k+1}}{2 z w} \\
& =1+\frac{1}{2} \sum_{m=1}^{\infty} \sum_{\alpha+\beta=m+2}\left(a_{\alpha, \beta-1}+b_{\beta, \alpha-1}\right) e^{i(\alpha-\beta) \theta} r^{m}
\end{aligned}
$$

According to the relation between systems (4) and (6), in fact transformation (9) can be regarded as the following real polar coordinate transformation of $\left.(4)\right|_{\delta=0}$ :

$$
x=r \cos \theta, \quad y=r \sin \theta .
$$

Under transformation (11), from (10) we have

$$
\frac{d r}{d \theta}=\frac{(i r / 2) \sum_{m=1}^{\infty} \sum_{\alpha+\beta=m+2}\left(a_{\alpha, \beta-1}-b_{\beta, \alpha-1}\right) e^{i(\alpha-\beta) \theta} r^{m}}{1+(1 / 2) \sum_{m=1}^{\infty} \sum_{\alpha+\beta=m+2}\left(a_{\alpha, \beta-1}+b_{\beta, \alpha-1}\right) e^{i(\alpha-\beta) \theta} r^{m}}
$$

For the complex constant $h,|h| \ll 1$, we write the solution of (12) associated with the initial condition $\left.r\right|_{\theta=0}=h$ as

$$
r=\tilde{r}(\theta, h)=h+\sum_{k=2}^{\infty} v_{k}(\theta) h^{k},
$$

in which $v_{2 k+1}(2 \pi)(k=1,2, \ldots)$ are called the $k$ th focal value of the origin of (4).

From (13), it is clear that the origin of (4) is a center if and only if all $v_{2 k+1}(2 \pi)=0(k=1,2, \ldots)$. Hence the computation of focal value plays an important role for settling the center problem. Liu and $\mathrm{Li}$ [19] gave some methods to compute focal values. Next we will introduce our method to calculate focal value through the following three lemmas.

Lemma 1 (see $[20,21])$. For system (6), one can derive successively the terms of the following formal series:

$$
M=1+\sum_{\alpha+\beta=1}^{\infty} c_{\alpha \beta} z^{\alpha} w^{\beta}
$$

such that

$$
\frac{\partial M}{\partial z} Z-\frac{\partial M}{\partial w} W+\left(\frac{\partial Z}{\partial z}-\frac{\partial W}{\partial w}\right) M=\sum_{m=1}^{\infty}(m+1) \mu_{m}(z w)^{m}
$$

where $c_{11}=1, c_{20}=c_{02}=0$, for all $c_{k k} \in R, k=2,3, \ldots$, and to any integer $m, \mu_{m}$ is determined by the following formulas:

$$
\begin{aligned}
& c_{1,1}=1, \quad c_{2,0}=c_{0,2}=0, \\
& \text { if }(\alpha=\beta=0, \beta \neq 1) \quad \text { or } \alpha<0, \quad \text { or } \beta<0, \\
& \text { then } c_{\alpha, \beta}=0,
\end{aligned}
$$

or else

$$
\begin{aligned}
c_{\alpha, \beta}= & \frac{1}{\beta-\alpha} \\
& \times \sum_{k+j=3}^{\alpha+\beta+2}\left[(\alpha-k+1) a_{k, j-1}\right. \\
& \left.\quad-(\beta-j+1) b_{j, k-1}\right] c_{\alpha-k+1, \beta-j+1},
\end{aligned}
$$

$$
\begin{aligned}
\mu_{m}=\sum_{k+j=3}^{2 m+2}[( & m-k+2) a_{k, j-1} \\
& \left.-(m-j+2) b_{j, k-1}\right] c_{m-k+2, m-j+2} .
\end{aligned}
$$

And $\mu_{k}$ in Lemma 1 is called $k$ th order singular point value at the origin of system (6).

Lemma 2 (see [20]). For system (4) and any positive integer $m$, among $v_{2 m}(2 \pi), v_{k}(2 \pi)$, and $v_{k}(\pi)$, there exists the following relation:

$$
\begin{aligned}
v_{2 m}(2 \pi)= & \frac{1}{1+v_{1}(\pi)} \\
& \times\left[\xi_{m}^{(0)}\left(v_{1}(2 \pi)-1\right)+\sum_{k=1}^{m-1} \xi_{m}^{(k)} v_{2 k+1}(2 \pi)\right],
\end{aligned}
$$

where $\xi_{m}^{(k)}$ are all polynomials of $v_{1}(\pi), v_{2}(\pi), \ldots, v_{m}(\pi)$ and $v_{1}(2 \pi), v_{2}(2 \pi), \ldots, v_{m}(2 \pi)$ with rational coefficients.

Obviously, We can imply that $v_{2 m}(2 \pi)=0$ when $v_{1}(2 \pi)=$ $1, v_{2 k+1}(2 \pi)=0, k=1,2, \ldots, m-1$.

Lemma 3 (see [20]). For system (4) $\left.\right|_{\delta=0},(6)$, and any positive integer $m$, the following assertion holds:

$$
v_{2 k+1}(2 \pi)=i \pi\left(\mu_{m}+\sum_{k=1}^{m-1} \xi_{m}^{(k)} \mu_{k}\right)
$$

where $\xi_{m}^{(k)},(k=1,2, \ldots, m-1)$ are polynomial functions of coefficients of system (6).

Obviously, the origin of system (4) is a center if and only if its all focal values vanish, namely, $v_{2 k+1}=0, k \in \mathbf{N}$. According to Lemmas 2 and 3, we have the following lemma.

Lemma 4 (see [20]). For systems (4) $\left.\right|_{\delta=0}$ and (6), the origin is a center if and only if the following relation holds:

$$
\mu_{m}(2 \pi)=0, \quad m \in \mathbf{N}
$$

Remark 5. In fact, Lemmas 1-4 have given a method to find original center condition of (4).

What is the isochronous center condition of the origin of (4) if the origin of (4) is a center? Next we introduce our method to obtain the isochronous center condition.

We denote that $\tau(\varphi, h)=\int_{0}^{\varphi}(d t / d \theta) d \theta$. From (10), we have

$$
\begin{aligned}
& \tau(\varphi, h) \\
& =\int_{0}^{\varphi} \frac{d t}{d \theta} d \theta
\end{aligned}
$$




$$
\begin{gathered}
\int_{0}^{\varphi}\left[1+\frac{1}{2} \sum_{m=1}^{\infty} \sum_{\alpha+\beta=m+2}\left(a_{\alpha, \beta-1}+b_{\beta, \alpha-1}\right)\right. \\
\left.\times e^{i(\alpha-\beta) \theta} \widetilde{r}^{m}(\theta, h)\right]^{-1} d \theta .
\end{gathered}
$$

Definition 6. For a sufficiently small complex constant $h$, the origin of system (6) is called a complex center if $\widetilde{r}(2 \pi, h) \equiv h$ of (13), and the origin is a complex isochronous center if

$$
\widetilde{r}(2 \pi, h) \equiv h, \quad \tau(2 \pi, h) \equiv 2 \pi .
$$

Lemma 7 (see [19]). For system (6), one can derive uniquely the formal series

$$
\xi=z+\sum_{k+j=2}^{\infty} c_{k j} z^{k} w^{j}, \quad \eta=w+\sum_{k+j=2}^{\infty} d_{k, j} w^{k} z^{j}
$$

where $c_{k+1, k}=d_{k+1, k}=0, k=1,2, \ldots, p_{k}$ and $q_{k}$ are polynomial in $a_{\alpha \beta}, b_{\alpha \beta}$ with rational coefficients, such that

$$
\frac{d \xi}{d T}=\xi+\sum_{j=1}^{\infty} p_{j} \xi^{j+1} \eta^{j}, \quad \frac{d \eta}{d T}=-\eta-\sum_{j=1}^{\infty} q_{j} \eta^{j+1} \xi^{j}
$$

Let $\mu_{0}=\tau_{0}=0, \mu_{k}=p_{k}-q_{k}, \tau_{k}=p_{k}+q_{k}, k=1,2, \ldots$, in which $\mu_{k}$ is $k$ th singular point value of the origin of system (6).

Definition 8 . For any positive integer $k$, one says that $\tau(k)=$ $p_{k}+q_{k}$ is the $k$ th complex period constant of origin of system (6).

Lemma 9 (see [19]). Suppose that the origin of system (6) is a complex center (i.e., all $\mu_{m}=0, m=1,2, \ldots$ ) and there exists a positive integer $k$, such that $\tau_{0}=\tau_{1}=\cdots=\tau_{k-1}=0, \tau_{k} \neq 0$; then

$$
\tau(2 \pi, h)=\pi\left[2-\tau_{k} h^{2 k}+o\left(h^{2 k}\right)\right]
$$

It is clear that the origin of system (6) is a complex isochronous center if and only if all $\mu_{k}=\tau_{k}=0, k=$ $1,2,3, \ldots$.

For the problem of the computation of $\tau_{k}$, [14] gives the following two theorems.

Theorem A (see [19]). For system (6), one can derive uniquely the formal series

$$
\begin{gathered}
f(z, w)=z+\sum_{k+j=2}^{\infty} c_{k j}^{\prime} z^{k} w^{j} \\
g(z, w)=w+\sum_{k+j=2}^{\infty} d_{k, j}^{\prime} w^{k} z^{j}
\end{gathered}
$$

where $c_{k+1, k}^{\prime}=d_{k+1, k}^{\prime}=0, k=1,2, \ldots$, such that

$$
\begin{aligned}
& \frac{d f}{d T}=f(z, w)+\sum_{j=1}^{\infty} p_{j}^{\prime} z^{j+1} w^{j}, \\
& \frac{d g}{d T}=-g(z, w)-\sum_{j=1}^{\infty} q_{j}^{\prime} w^{j+1} z^{j},
\end{aligned}
$$

and when $k-j-1 \neq 0, c_{k j}^{\prime}$ and $d_{k, j}^{\prime}$ are determined by the recursive formulas

$$
\begin{aligned}
c_{k j}^{\prime}= & \frac{1}{j+1-k} \\
& \times \sum_{\alpha+\beta=3}^{k+j+1}\left[(k-\alpha+1) a_{\alpha, \beta-1}\right. \\
& \left.-(j-\beta+1) b_{\beta, \alpha-1}\right] c_{k-\alpha+1, j-\beta+1}^{\prime}, \\
d_{k j}^{\prime}= & \frac{1}{j+1-k} \\
& \times \sum_{\alpha+\beta=3}^{k+j+1}\left[(k-\alpha+1) b_{\alpha, \beta-1}\right. \\
&
\end{aligned}
$$

and for any positive integer $j, p_{j}^{\prime}$, and $q_{j}^{\prime}$ are determined by the recursive formulas

$$
\begin{aligned}
& p_{j}^{\prime}=\sum_{\alpha+\beta=3}^{2 j+2}[(j-\alpha+2) a_{\alpha, \beta-1} \\
&\left.-(j-\beta+1) b_{\beta, \alpha-1}\right] c_{j-\alpha+2, j-\beta+1}^{\prime}, \\
& q_{j}^{\prime}=\sum_{\alpha+\beta=3}^{2 j+2}\left[(j-\alpha+2) b_{\alpha, \beta-1}\right. \\
&\left.\quad-(j-\beta+1) a_{\beta, \alpha-1}\right] d_{j-\alpha+2, j-\beta+1}^{\prime} .
\end{aligned}
$$

In (28) and (29), we have taken $c_{1,0}^{\prime}=d_{1,0}^{\prime}=1, c_{0,1}^{\prime}=d_{0,1}^{\prime}=0$, and if $\alpha<0$ or $\beta<0$, we take $a_{\alpha \beta}=b_{\alpha \beta}=c_{\alpha \beta}^{\prime}=d_{\alpha \beta}^{\prime}=0$.

Theorem B (see [19]). Let $p_{0}=q_{0}=p_{0}^{\prime}=q_{0}^{\prime}=0$. If there is a positive integer $m$, such that

$$
p_{0}=q_{0}=p_{1}=q_{1}=\cdots=p_{m-1}=q_{m-1}=0,
$$

then

$$
\begin{gathered}
p_{0}^{\prime}=q_{0}^{\prime}=p_{1}^{\prime}=q_{1}^{\prime}=\cdots=p_{m-1}^{\prime}=q_{m-1}^{\prime}=0, \\
p_{m}=p_{m}^{\prime}, \quad q_{m}=q_{m}^{\prime},
\end{gathered}
$$

and vice versa.

Actually, Lemma 7 and the above two theorems give an algorithm to compute $\tau_{m}$. For any positive integer $m$, in 
order to compute $\tau_{m}$, we only need to carry out the addition, subtraction, multiplication, and division to the coefficients of system (6). The algorithm is recursive. It avoids some complicated integrating operations and solving equations. In addition, it can be easily realized by computer algebra systems such as Mathematica.

Notice that the complex period constants are polynomials of the coefficients of system (6). According to the Hilbert basis theorem, there exists $m \in \mathbf{N}$ such that all $\tau_{k}=0(k=1,2, \ldots)$ if and only if $\tau_{1}=\tau_{2}=\cdots=\tau_{m}=0$. We say that the set $\left\{\tau_{1}, \tau_{2}, \ldots, \tau_{m}\right\}$ is a period constant basis of system (6). To determine isochronous center of a system, the key idea is to find a period constant basis.

Remark 10. Lemma 7 and Theorems A and B offer a method to find a necessary condition of isochronicity.

\section{The Reduction and Bicenter Condition of System (2)}

After introducing the method to calculate the focal values and period constants of system (4), we try to make some appropriate transformations so as to carry out our investigation about system (2).

By means of Bendixson homeomorphous transformation

$$
u=\frac{x}{x^{2}+y^{2}}, \quad v=\frac{y}{x^{2}+y^{2}},
$$

and time transformation

$$
d t=\left(x^{2}+y^{2}\right)^{3} d \tau
$$

system (2) can be transformed into the following real system:

$$
\begin{gathered}
\frac{d u}{d \tau}=\delta u-v+\frac{3 \delta}{2} u^{2}+2 a_{1} u v+\frac{\delta}{2} u^{3}+a_{1} u^{2} v+a_{3} v^{3} \\
\frac{d v}{d \tau}=u+\delta v+\frac{3}{2} u^{2}+2 a_{4} u v+a_{5} v^{2}+\frac{1}{2} u^{3} \\
+a_{4} u^{2} v+a_{5} u v^{2}+a_{6} v^{3}
\end{gathered}
$$

After making the above two transformations, the infinity and the elementary focus point $(-1 / 2,0)$ of $(2)$, respectively, become the origin and $(-2,0)$ of system $(34)$. For system (34), we have the following theorem.

Theorem 11. System (34) is a class of $z_{2}$-equivariant cubic systems about point $(-1,0)$.

Proof. By means of translation transformation

$$
u=x-1, \quad v=y,
$$

system (34) turns into the following system:

$$
\begin{aligned}
& \frac{d x}{d \tau}=-\frac{\delta}{2} x-\left(a_{1}+1\right) y+\frac{\delta}{2} x^{3}+a_{1} x^{2} y+a_{3} y^{3}, \\
& \frac{d y}{d \tau}+\left(\delta-a_{4}\right) y+\frac{1}{2} x^{3}+a_{4} x^{2} y+a_{5} x y^{2}+a_{6} y^{3} .
\end{aligned}
$$

Obviously point $(-x,-y)$ satisfies $(36)$ if $(x, y)$ satisfies (36); then system (36) is a class of $z_{2}$-equivariant cubic systems about the origin. Hence system (34) is a class of $z_{2}$-equivariant cubic systems about point $(-1,0)$. Proof ends.

After making transformations (32) and (33), system (2) becomes a symmetric system about point $(-1,0)$ (i.e., system (34)), so here we call system (2) a class of quasi symmetric systems. In fact, through investigating the center condition of the origin of system (34), those of the infinity and the elementary focus point $(-1 / 2,0)$ of $(2)$ can been forecasted.

In order to investigate the centers problem of (2), we may as well study system (34) or system (36).

Making the transformation

$$
z=u+i v, \quad w=u-i v, \quad T=i \tau, \quad i=\sqrt{-1},
$$

system $\left.(34)\right|_{\delta}=0$ becomes

$$
\begin{gathered}
\frac{d z}{d T}=z+Z_{2}(z, w)+Z_{3}(z, w), \\
\frac{d w}{d T}=-w-W_{2}(z, w)-W_{3}(z, w),
\end{gathered}
$$

in which

$$
\begin{aligned}
& Z_{2}(z, w)=\frac{1}{8}\left(3-4 a_{1}-4 i a_{4}-2 a_{5}\right) z^{2}+\frac{1}{4}\left(3+2 a_{5}\right) z w \\
& +\frac{1}{8}\left(3+4 a_{1}+4 i a_{4}-2 a_{5}\right) w^{2} \\
& Z_{3}(z, w)=\frac{1}{16}\left(1-2 a_{1}+2 a_{3}-2 i a_{4}-2 a_{5}+2 i a_{6}\right) z^{3} \\
& +\frac{1}{16}\left(3-2 a_{1}-6 a_{3}-2 i a_{4}+2 a_{5}-6 i a_{6}\right) z^{2} w \\
& +\frac{1}{16}\left(3+2 a_{1}+6 a_{3}+2 i a_{4}+2 a_{5}+6 i a_{6}\right) z w^{2} \\
& +\frac{1}{16}\left(1+2 a_{1}-2 a_{3}+2 i a_{4}-2 a_{5}-2 i a_{6}\right) w^{3}, \\
& W_{2}(z, w)=\frac{1}{8}\left(3-4 a_{1}+4 i a_{4}+2 a_{5}\right) w^{2}+\frac{1}{4}\left(3+2 a_{5}\right) z w \\
& +\frac{1}{8}\left(3+4 a_{1}-4 i a_{4}-2 a_{5}\right) w^{2} \\
& W_{3}(z, w)=\frac{1}{16}\left(1+2 a_{1}-2 a_{3}-2 i a_{4}-2 a_{5}+2 i a_{6}\right) z^{3} \\
& +\frac{1}{16}\left(3+2 a_{1}+6 a_{3}-2 i a_{4}+2 a_{5}-6 i a_{6}\right) z^{2} w \\
& +\frac{1}{16}\left(3-2 a_{1}-6 a_{3}+2 i a_{4}+2 a_{5}+6 i a_{6}\right) z w^{2} \\
& +\frac{1}{16}\left(1-2 a_{1}+2 a_{3}+2 i a_{4}-2 a_{5}-2 i a_{6}\right) w^{3} .
\end{aligned}
$$


System (38) is called the complex concomitant system of system (34). Clearly system (38) belongs to the class of system (6), so we can use the formulas of Lemma 1 and the conclusion of Lemma 4 to compute and simplify the singular points values by using computational software such as Mathematica; we obtain the following theorem.

Theorem 12. The first 5 singular points' values of the origin for (38) are as follows:

$$
\begin{gathered}
\mu_{1}=\frac{i}{4}\left(2 a_{4}+2 a_{4} a_{5}-3 a_{6}\right) ; \\
\mu_{2}=\frac{i}{36} a_{4}\left[\left(1+a_{5}\right)\left(18+18 a_{1}+8 a_{4}^{2}\right)\right. \\
\left.-a_{3}\left(9+12 a_{1}+12 a_{5}\right)\right] ;
\end{gathered}
$$

(1) if $a_{5}=-1$, then

$$
\begin{gathered}
\mu_{2}=-\frac{i}{12} a_{3} a_{4}\left(4 a_{1}-1\right) ; \\
\mu_{3}=\frac{i}{384} a_{3} a_{4}\left(84 a_{3}-16 a_{4}^{2}-45\right) ; \\
\mu_{4}=\frac{i}{169344} a_{4}\left(3+2 a_{4}^{2}\right)\left(45+16 a_{4}^{2}\right)^{2} ;
\end{gathered}
$$

(2) if $a_{5} \neq-1$, then

$$
\begin{gathered}
\mu_{3}=\frac{i a_{3} a_{4}}{96\left(1+a_{5}\right)}\left(4 a_{1}-10 a_{5}-11\right) h_{0} ; \\
\mu_{4}=-\frac{7 i a_{3} a_{4}}{1500}\left(1+a_{1}-5 a_{3}\right) \\
\times\left(29 a_{1}^{2}-a_{1}-2+28 a_{1}^{3}+45 a_{3}-105 a_{1} a_{3}\right) ; \\
\mu_{5}=\frac{7 i}{36} a_{3}^{4} a_{4}\left(11-76 a_{3}+140 a_{3}^{2}\right)
\end{gathered}
$$

in which

$$
\begin{aligned}
h_{0}= & -6-10 a_{1}-4 a_{1}^{2}+3 a_{3}+6 a_{1} a_{3}-10 a_{5} \\
& -14 a_{1} a_{5}-4 a_{1}^{2} a_{5}+6 a_{3} a_{5}-4 a_{5}^{2}-4 a_{1} a_{5}^{2} .
\end{aligned}
$$

In the above expressions of $\mu_{k}$, one lets $\mu_{i}=0, i=1,2, \ldots, k-1$.

Proof. According to Lemma 1, we have

$$
\mu_{1}=\frac{i}{4}\left(2 a_{4}+2 a_{4} a_{5}-3 a_{6}\right) .
$$

Let $a_{6}=(2 / 3) a_{4}\left(1+a_{5}\right)$; then

$$
\begin{gathered}
\mu_{1}=0 ; \\
\mu_{2}=\frac{i}{36} a_{4}\left[\left(1+a_{5}\right)\left(18+18 a_{1}+8 a_{4}^{2}\right)\right. \\
\left.-a_{3}\left(9+12 a_{1}+12 a_{5}\right)\right] .
\end{gathered}
$$

(1) If $a_{5}=-1$, then

$$
\mu_{2}=-\frac{i}{12} a_{3} a_{4}\left(4 a_{1}-1\right) ;
$$

if $a_{3} a_{4}=0$, from Lemma 1 , we obtain that $\mu_{k}=0, k=$ $2,3,4,5$. Hence let $a_{1}=1 / 4$; then

$$
\mu_{3}=\frac{i}{384} a_{3} a_{4}\left(84 a_{3}-16 a_{4}^{2}-45\right) .
$$

Moreover let $a_{3}=(1 / 84)\left(45+16 a_{4}^{2}\right)$; then

$$
\mu_{3}=0, \quad \mu_{4}=\frac{i}{169344} a_{4}\left(3+2 a_{4}^{2}\right)\left(45+16 a_{4}^{2}\right)^{2} ;
$$

while if $a_{4} \neq 0$, then $\mu_{4} \neq 0$; at this time only four singular point values can be obtained.

(2) If $a_{5} \neq-1$, letting

$$
a_{4}^{2}=\frac{3\left(4 a_{3} a_{5}-6 a_{1} a_{5}-6 a_{5}+4 a_{1} a_{3}+3 a_{3}-6 a_{1}-6\right)}{\left(8+8 a_{5}\right)},
$$

then

$$
\mu_{2}=0 ; \quad \mu_{3}=\frac{i a_{3} a_{4}}{96\left(1+a_{5}\right)}\left(4 a_{1}-10 a_{5}-11\right) h_{0} .
$$

Because $a_{3} a_{4} h_{0}=0$ will induce $\mu_{3}=\mu_{4}=\mu_{5}=0$, let $a_{5}=$ $(1 / 10)\left(4 a_{1}-11\right)$; at this time

$$
\begin{aligned}
\mu_{4}= & -\frac{7 i a_{3} a_{4}}{1500}\left(1+a_{1}-5 a_{3}\right) \\
& \times\left(29 a_{1}^{2}-a_{1}-2+28 a_{1}^{3}+45 a_{3}-105 a_{1} a_{3}\right),
\end{aligned}
$$

while $a_{3} a_{4}\left(29 a_{1}^{2}-a_{1}-2+28 a_{1}^{3}+45 a_{3}-105 a_{1} a_{3}\right)=0$ will induce $\mu_{4}=\mu_{5}=0$, let $a_{1}=5 a_{3}-1$; then

$$
\mu_{5}=\frac{7 i}{36} a_{3}^{4} a_{4}\left(11-76 a_{3}+140 a_{3}^{2}\right) \text {. }
$$

Proof ends.

Remark. The equation $11-76 a_{3}+140 a_{3}^{2}=0$ has not real roots, so only five singular point values exist.

From Theorem 12 and Lemma 4, we have the following theorem.

Theorem 13. The first 5 focal values of the origin of (21) (or the first 5 general focal values of the infinity and the elementary focus $(-1 / 2,0)$ of $(4))$ are as follows:

$$
\begin{gathered}
v_{3}=-\frac{\pi}{4}\left(2 a_{4}+2 a_{4} a_{5}-3 a_{6}\right) ; \\
v_{5}=-\frac{\pi}{36} a_{4}\left[\left(1+a_{5}\right)\left(18+18 a_{1}+8 a_{4}^{2}\right)\right. \\
\left.-a_{3}\left(9+12 a_{1}+12 a_{5}\right)\right] .
\end{gathered}
$$

(1) If $a_{5}=-1$, then

$$
\begin{gathered}
v_{5}=\frac{\pi}{12} a_{3} a_{4}\left(4 a_{1}-1\right) ; \\
v_{7}=-\frac{\pi}{384} a_{3} a_{4}\left(84 a_{3}-16 a_{4}^{2}-45\right) . \\
v_{9}=-\frac{\pi}{169344} a_{4}\left(3+2 a_{4}^{2}\right)\left(45+16 a_{4}^{2}\right)^{2} .
\end{gathered}
$$


(2) If $a_{5} \neq-1$, then

$$
\begin{gathered}
v_{7}=-\frac{\pi a_{3} a_{4}}{96\left(1+a_{5}\right)}\left(4 a_{1}-10 a_{5}-11\right) h_{0}, \\
v_{9}=\frac{7 \pi a_{3} a_{4}}{1500}\left(1+a_{1}-5 a_{3}\right) \\
\times\left(29 a_{1}^{2}-a_{1}-2+28 a_{1}^{3}+45 a_{3}-105 a_{1} a_{3}\right), \\
v_{11}=-\frac{7 \pi}{36} a_{3}^{4} a_{4}\left(11-76 a_{3}+140 a_{3}^{2}\right) .
\end{gathered}
$$

in which

$$
\begin{aligned}
h_{0}= & -6-10 a_{1}-4 a_{1}^{2}+3 a_{3}+6 a_{1} a_{3}-10 a_{5} \\
& -14 a_{1} a_{5}-4 a_{1}^{2} a_{5}+6 a_{3} a_{5}-4 a_{5}^{2}-4 a_{1} a_{5}^{2} .
\end{aligned}
$$

In the above expressions of $v_{2 k+1}$, we have let $v_{2 i-1}=0, i=$ $2, \ldots, k$. theorem.

According to Theorem 13, it is easy to obtain the following

Theorem 14. The first 5 focal values of the origin of (34) (or the first 5 focal values of the infinity and the elementary focus $(-1 / 2,0)$ of $(2))$ vanish if and only if one of the following conditions holds:

$$
\begin{array}{ll}
\left(H_{1}\right) & a_{4}=a_{6}=0 \\
\left(H_{2}\right) & a_{4}^{2}=-(9 / 4)\left(1+a_{1}\right)>0, a_{3}=0, a_{6}=(2 / 3) a_{4}\left(1+a_{5}\right), \\
& a_{5} \neq-1 \\
\left(H_{3}\right) \quad a_{6}=(2 / 3) a_{4}\left(1+a_{5}\right), a_{5} \neq-1, a_{3} \neq 0, a_{4}^{2}=3\left(4 a_{3} a_{5}-\right. \\
\\
\left.6 a_{1} a_{5}-6 a_{5}+4 a_{1} a_{3}+3 a_{3}-6 a_{1}-6\right) /\left(8+8 a_{5}\right)>0 \\
h_{0}=0
\end{array}
$$

Theorem 15. The origin of (34) is a center (the infinity and the elementary focus $(-1 / 2,0)$ of $(2)$ are two centers), if and only if one of the conditions $\left(\mathrm{H}_{1}\right),\left(\mathrm{H}_{2}\right)$, and $\left(\mathrm{H}_{3}\right)$ holds.

Proof. (1) According to Theorem 14, if the origin of (34) is a center (the infinity and the elementary focus $(-1 / 2,0)$ of (2) are two general centers), then one of the conditions $\left(H_{1}\right)$, $\left(\mathrm{H}_{2}\right)$, and $\left(\mathrm{H}_{3}\right)$ holds. So necessary condition is correct.

(2) Next we prove sufficient condition.

(2.1) If $\left(H_{1}\right)$ holds, then (36) $\left.\right|_{\delta=0}$ becomes

$$
\begin{gathered}
\frac{d x}{d \tau}=-\left(a_{1}+1\right) y+a_{1} x^{2} y+a_{3} y^{3} . \\
\frac{d y}{d \tau}=-\frac{1}{2} x+\frac{1}{2} x^{3}+a_{5} x y^{2}
\end{gathered}
$$

Letting

$$
\begin{gathered}
g_{1}(x, y)=2+a_{1}+a_{5}-a_{1} x^{2}-a_{5} x^{2}-2 a_{3} y^{2} \\
+2 a_{1} a_{5} y^{2}-2 a_{5}^{2} y^{2} \\
g_{2}(x, y)=-1+x^{2}+2 a_{5} y^{2}
\end{gathered}
$$

then system (57) has an integral factor $M_{1}(x, y)=f_{1}^{-1}$ and a first integral $F_{1}(x, y)=f_{1} f_{2}^{\left(a_{1}+a_{5}\right)}$, in which

$$
\begin{aligned}
& f_{1}=g_{1}^{2}-\left[2 a_{3}+\left(a_{1}-a_{5}\right)^{2}\right] g_{2}^{2},
\end{aligned}
$$

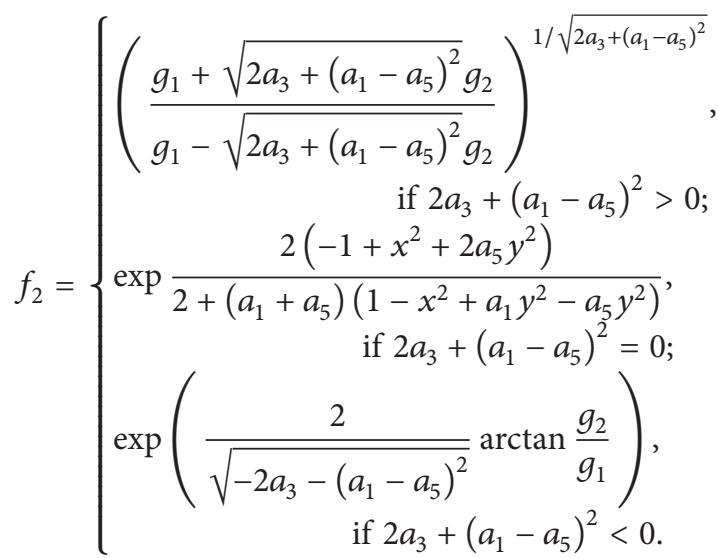

Considering that system (34) can turn into system (36) by making transformations, hence the origin of (34) is a center when $\left(H_{1}\right)$ holds; moreover, the infinity and the elementary focus $(-1 / 2,0)$ of $(2)$ are two centers.

(2.2) If $\left(\mathrm{H}_{2}\right)$ holds, then (36) $\left.\right|_{\delta=0}$ becomes

$$
\begin{gathered}
\frac{d x}{d \tau}=\frac{4}{9} a_{4}^{2} y-\frac{1}{9}\left(9+4 a_{4}^{2}\right) x^{2} y, \\
\frac{d y}{d \tau}=-\frac{1}{2} x-a_{4} y+\frac{1}{2} x^{3}+a_{4} x^{2} y+a_{5} x y^{2} \\
+\frac{2}{3} a_{4}\left(1+a_{5}\right) y^{3},
\end{gathered}
$$

while system (61) has an integral factor

$$
M_{2}(x, y)=f_{3}^{-3} f_{4}^{\left(9-8 a_{4}^{2}+18 a_{5}\right) / 2}
$$

and a first integral

$$
\begin{aligned}
F_{2}= & \left(3 x+4 a_{4} y\right) f_{3}^{-2} f_{4}^{9\left(3+2 a_{5}\right) / 2} \\
& -6\left(1+a_{5}\right) \int \frac{f_{4}^{9\left(3+2 a_{5}\right) / 2} d x}{9+\left(9+4 a_{4}^{2}\right)\left(-1+x^{2}\right)},
\end{aligned}
$$

in which

$$
\begin{gathered}
f_{3}=3 x+2 a_{4} y, \\
f_{4}= \begin{cases}{\left[9+\left(9+4 a_{4}^{2}\right)\left(-1+x^{2}\right)\right]^{1 /\left(9+4 a_{4}^{2}\right)},} & \text { if } 9+4 a_{4}^{2} \neq 0 ; \\
e^{\left(-1+x^{2}\right) / 9}, & \text { if } 9+4 a_{4}^{2}=0 .\end{cases}
\end{gathered}
$$

Considering that system (34) can turn into system (36) by making transformations, hence the origin of (34) is a center when $\left(\mathrm{H}_{2}\right)$ holds; moreover, the infinity and the elementary focus $(-1 / 2,0)$ of $(2)$ are two centers. 
(2.3) If $\left(\mathrm{H}_{3}\right)$ holds, then (34) $\left.\right|_{\delta=0}$ has an integral factor $M_{3}(x, y)=f_{5}^{2\left(a_{1}+a_{5}\right)}$ and a first integral $F_{3}(x, y)=$ $f_{5}^{\left(1+2 a_{1}+2 a_{5}\right)} f_{6}$, in which

$$
\begin{gathered}
f_{5}=\left(a_{1}+a_{5}\right) x-a_{4} y, \\
f_{6}=\left(2+a_{1}+a_{5}\right)\left[a_{4} x+2\left(1+a_{1}\right)\left(a_{1}+a_{5}\right) y\right] \\
-\left(1+a_{1}+a_{5}\right) \\
\times\left[a_{4} x^{3}+2\left(1+a_{1}\right)\left(a_{1}+a_{5}\right) x^{2} y\right. \\
\left.+2 a_{4}\left(1+a_{5}\right) x y^{2}+2 a_{3}\left(a_{1}+a_{5}\right) y^{3}\right] .
\end{gathered}
$$

Considering that system (34) can turn into system (36) by making transformations, hence the origin of (34) is a center when $\left(\mathrm{H}_{3}\right)$ holds; moreover, the infinity and the elementary focus $(-1 / 2,0)$ of $(2)$ are two centers. Proof ends.

\section{Isochronous Center Condition of $(-1 / 2,0)$ of System (2)}

After obtaining the conditions that the infinity and the elementary focus $(-1 / 2,0)$ are two centers, next we continue to investigate isochronicity of the elementary focus $(-1 / 2,0)$ according to three different cases (i.e., $\left(H_{1}\right),\left(H_{2}\right)$, and $\left(H_{3}\right)$ ) in order.

4.1. The Isochronicity of Case $\left(H_{1}\right)$. If condition $\left(H_{1}\right)$ holds, then system $\left.(34)\right|_{\delta=0}$ becomes

$$
\begin{gathered}
\frac{d u}{d \tau}=-v+2 a_{1} u v+a_{1} u^{2} v+a_{3} v^{3}, \\
\frac{d v}{d \tau}=u+\frac{3}{2} u^{2}+a_{5} v^{2}+\frac{1}{2} u^{3}+a_{5} u v^{2} .
\end{gathered}
$$

System (66) complex concomitant system is as follows:

$$
\begin{gathered}
\frac{d z}{d T}=z+Z_{2}(z, w)+Z_{3}(z, w) \\
\frac{d w}{d T}=-w-W_{2}(z, w)-W_{3}(z, w)
\end{gathered}
$$

in which

$$
\begin{aligned}
Z_{2}(z, w)= & \frac{1}{8}\left(3-4 a_{1}-2 a_{5}\right) z^{2}+\frac{1}{4}\left(3+2 a_{5}\right) z w \\
& +\frac{1}{8}\left(3+4 a_{1}-2 a_{5}\right) w^{2} \\
Z_{3}(z, w)= & \frac{1}{16}\left(1-2 a_{1}+2 a_{3}-2 a_{5}\right) z^{3} \\
& +\frac{1}{16}\left(3-2 a_{1}-6 a_{3}+2 a_{5}\right) z^{2} w \\
& +\frac{1}{16}\left(3+2 a_{1}+6 a_{3}+2 a_{5}\right) z w^{2} \\
& +\frac{1}{16}\left(1+2 a_{1}-2 a_{3}-2 a_{5}\right) w^{3}
\end{aligned}
$$

$$
\begin{aligned}
W_{2}(z, w)= & \frac{1}{8}\left(3-4 a_{1}+2 a_{5}\right) w^{2}+\frac{1}{4}\left(3+2 a_{5}\right) z w \\
& +\frac{1}{8}\left(3+4 a_{1}-2 a_{5}\right) w^{2} \\
W_{3}(z, w)= & \frac{1}{16}\left(1+2 a_{1}-2 a_{3}-2 a_{5}\right) z^{3} \\
& +\frac{1}{16}\left(3+2 a_{1}+6 a_{3}+2 a_{5}\right) z^{2} w \\
& +\frac{1}{16}\left(3-2 a_{1}-6 a_{3}+2 a_{5}\right) z w^{2} \\
& +\frac{1}{16}\left(1-2 a_{1}+2 a_{3}-2 a_{5}\right) w^{3}
\end{aligned}
$$

According to formulas (28) and (29) of Theorem A and $\tau_{k}=p_{k}+q_{k}=p_{k}^{\prime}+q_{k}^{\prime}$, we can compute periodic constants of systems (66) and (67).

Theorem 16. The first four period constants of the origin of system (66) or (67) are as follows:

$$
\begin{gathered}
\tau_{1}=\frac{1}{12}\left(-18-4 a_{1}^{2}-9 a_{3}-12 a_{5}+10 a_{1} a_{5}-4 a_{5}^{2}\right), \\
\tau_{2}=\frac{1}{18}\left(-108-45 a_{1}-12 a_{1}^{2}-117 a_{5}-24 a_{1} a_{5}\right. \\
\left.-2 a_{1}^{2} a_{5}-36 a_{5}^{2}-4 a_{1} a_{5}^{2}-2 a_{5}^{2}\right) \\
\tau_{3}=\frac{1}{1080} h_{1}, \\
\tau_{4}=-\frac{1}{1782606477375000000}\left(9+a_{5}\right)\left(3+2 a_{5}\right)^{2} h_{2} .
\end{gathered}
$$

in which

$$
\begin{aligned}
h_{1}= & \left(9720+4050 a_{1}+27 a_{1}^{2}-300 a_{1}^{3}-100 a_{1}^{4}\right. \\
& +8802 a_{5}+1134 a_{1} a_{5}-1416 a_{1}^{2} a_{5} \\
& \left.-200 a_{1}^{3} a_{5}+2115 a_{5}^{2}+468 a_{1} a_{5}^{2}-100 a_{1}^{2} a_{5}^{2}\right), \\
h_{2}= & 441227872054417689+969414967442849922 a_{5} \\
+ & 729276873221792484 a_{5}^{2}+232198402133252079 a_{5}^{3} \\
+ & 40172927013408528 a_{5}^{4}+3428874437224704 a_{5}^{5} \\
+ & 49018338325504 a_{5}^{6},
\end{aligned}
$$

In the above expression of $\tau_{m}$, we have let $\tau_{1}=\cdots \tau_{m-1}=0$, $m=2,3,4$.

Theorem 17. The first four period constants of the origin of system (66) or (67) are zero, if and only if one of the following two conditions holds:

$$
\left(C_{1}\right) a_{1}=a_{5}=-3 / 2, a_{3}=1 / 2 ;
$$




$$
\left(C_{2}\right) a_{1}=-3, a_{3}=0, a_{5}=-9 .
$$

Proof. By computing the resultant of $\tau_{2}$ and $\tau_{3}$ about variable $a_{1}$, we have

$$
\text { Resultant }\left[\tau_{3}, \tau_{2}, a_{1}\right]=\frac{1}{212576400}\left(9+a_{5}\right)\left(3+2 a_{5}\right)^{2} R,
$$

in which

$$
\begin{aligned}
R= & 4214349+14258511 a_{5}+18779526 a_{5}^{2}+12158343 a_{5}^{3} \\
& +4086747 a_{5}^{4}+727632 a_{5}^{5}+69888 a_{5}^{6}+1024 a_{5}^{7} .
\end{aligned}
$$

Also the resultant of $R$ and $h_{2}$ about $a_{5}$ is as follows:

$$
\text { Resultant }\left[R, h_{2}, a_{5}\right]=-28488259 \cdots \neq 0 \text {, }
$$

which shows that $R=0$ cannot deduce $\tau_{2}=\tau_{3}=\tau_{4}=0$.

From (71), let $\tau_{2}=\tau_{3}=\tau_{4}=0$; then Resultant $\left[\tau_{3}, \tau_{2}, a_{1}\right]=0$; hence $a_{5}=-3 / 2$ or $a_{5}=-9$.

If $a_{5}=-3 / 2$, then $\tau_{4}=0$; continue to let $\tau_{3}=0$ (i.e., $h_{1}=0$ ); then $a_{1}=-3 / 2$; next let $\tau_{1}=0$; then $a_{3}=1 / 2$. Hence condition $\left(C_{1}\right)$ holds. At the same time, if condition $\left(C_{1}\right)$ holds, it is easy to obtain that $\tau_{1}=\tau_{2}=\tau_{3}=\tau_{4}=0$.

If $a_{5}=-9$, then $\tau_{4}=0$; continue to let $\tau_{3}=0$ (i.e., $h_{1}=0$ ); then $a_{1}=-3$; next let $\tau_{1}=0$; then $a_{3}=0$. Hence condition $\left(C_{2}\right)$ holds. At the same time, if condition $\left(C_{2}\right)$ holds, it is easy to obtain that $\tau_{1}=\tau_{2}=\tau_{3}=\tau_{4}=0$.

Clearly, condition $\left(C_{1}\right)$ or $\left(C_{2}\right)$ is necessary for the isochronicity of the origin of system (66) or (67). Moreover, we will prove that condition $\left(C_{1}\right)$ or $\left(C_{2}\right)$ is sufficient condition for the isochronicity of the origin of system (66) or (67).

Theorem 18. The origin of system (66) or (67) is an isochronous center if and only if one of $\left(C_{1}\right)$ and $\left(C_{2}\right)$ holds.

Proof. If the origin of system (66) or (67) is an isochronous center, according to Theorem 17 , then one of $\left(C_{1}\right)$ and $\left(C_{2}\right)$ holds. Hence, the necessary condition is proved.

Next we will prove the sufficient condition.

(1) If $\left(C_{1}\right)$ holds, then system (66) becomes

$$
\begin{aligned}
& \frac{d u}{d \tau}=-\frac{1}{2} v\left(2+6 u+3 u^{2}-v^{2}\right), \\
& \frac{d v}{d \tau}=\frac{1}{2}(1+u)\left(2 u+u^{2}-3 v^{2}\right) .
\end{aligned}
$$

For (74), make the following analytic polar transformations:

$$
\begin{gathered}
u_{1}=\frac{2 u+5 u^{2}+4 u^{3}+u^{4}+3 v^{2}+4 u v^{2}+2 u^{2} v^{2}+v^{4}}{2\left(1+2 u+u^{2}+v^{2}\right)^{2}} \\
v_{1}=\frac{(1+u) v}{\left(1+2 u+u^{2}+v^{2}\right)^{2}}
\end{gathered}
$$

which satisfy $d u_{1} / d \tau=u_{1}, d v_{1} / d \tau=-v_{1}$, so the origin of system (74) is an isochronous center.

(2) If $\left(C_{2}\right)$ holds, then system (66) becomes

$$
\begin{gathered}
\frac{d u}{d \tau}=-v-6 u v-3 u^{2} v \\
\frac{d v}{d \tau}=u+\frac{3}{2} u^{2}-9 v^{2}+\frac{1}{2} u^{3}-9 u v^{2}
\end{gathered}
$$

For (76), make the following analytic polar transformations:

$$
\begin{gathered}
u_{2}=\frac{u(u-1)(u-2)}{\sqrt{2}\left(3 u-(3 / 2) u^{2}-1 / 2\right)^{3 / 2}}, \\
v_{2}=\frac{\sqrt{2} v}{\left(3 u-(3 / 2) u^{2}-1 / 2\right)^{3 / 2}},
\end{gathered}
$$

which satisfy $d u_{2} / d \tau=u_{2}, d v_{2} / d \tau=-v_{2}$, so the origin of system (76) is an isochronous center.

Considering that system (34) is a class of $z_{2}$-equivariant cubic systems about point $(-1,0)$, we have the following Theorem.

Theorem 19. If one of the conditions $\left(C_{1}\right)$ and $\left(C_{2}\right)$ holds under condition $\left(H_{1}\right)$, then the origin and point $(-2,0)$ of system $(34)$ are two isochronous centers.

Considering that system (2) can be changed into system (34) under the transformations (32) and (33), ulteriorly, we have the following Theorem.

Theorem 20. If one of the conditions $\left(C_{1}\right)$ and $\left(C_{2}\right)$ holds under condition $\left(H_{1}\right)$, then the singular point $(-1 / 2,0)$ of system (2) is an isochronous center.

4.2. The Isochronicity of Case $\left(\mathrm{H}_{2}\right)$. If condition $\left(\mathrm{H}_{2}\right)$ holds, then system (34) $\left.\right|_{\delta=0}$ becomes

$$
\begin{aligned}
& \frac{d u}{d \tau}=- \frac{1}{9} v\left(9+18 u+8 a_{4}^{2} u+9 u^{2}+4 a_{4}^{2} u^{2}\right), \\
& \frac{d v}{d \tau}=\frac{1}{6}\left(6 u+9 u^{2}+3 u^{3}+12 a_{4} u v+6 a_{4} u^{2} v\right. \\
&\left.+6 a_{5} v^{2}+6 a_{5} u v^{2}+4 a_{4} v^{3}+4 a_{4} a_{5} v^{3}\right) .
\end{aligned}
$$

System (78) complex concomitant system belongs to the form of (38). Hence we can use formulas (28) and (29) of Theorem $\mathrm{A}$ and $\tau_{k}=p_{k}+q_{k}=p_{k}^{\prime}+q_{k}^{\prime}$ to compute periodic constants of system (78).

Theorem 21. The first three period constants of the origin of system (78) are as follows:

$$
\begin{aligned}
\tau_{1}=\frac{1}{486}( & -891-306 a_{4}^{2}-32 a_{4}^{4}-891 a_{5} \\
- & \left.180 a_{4}^{2} a_{5}-162 a_{5}^{2}\right),
\end{aligned}
$$




$$
\begin{aligned}
& \tau_{2}= \frac{1}{2916}\left(9+4 a_{4}^{2}\right) \\
& \times\left(729+234 a_{4}^{2}+32 a_{4}^{4}+567 a_{5}+108 a_{4}^{2} a_{5}\right), \\
& \tau_{3}=-\frac{1}{3346110}\left(9+a_{4}^{2}\right)\left(9+4 a_{4}^{2}\right)\left(999+165 a_{4}^{2}+16 a_{4}^{4}\right) .
\end{aligned}
$$

In the above expression of $\tau_{m}$, we have let $\tau_{1}=\cdots \tau_{m-1}=0$, $m=2,3$.

From Theorem 21, $\tau_{3}=0$ has not real number roots. Hence under condition $\left(\mathrm{H}_{2}\right)$, the origin of system (78) cannot become an isochronous center. Accordingly, the singular point $(-1 / 2,0)$ of system (2) cannot become an isochronous center under condition $\left(\mathrm{H}_{2}\right)$.

4.3. The Isochronicity of Case $\left(\mathrm{H}_{3}\right)$. If condition $\left(\mathrm{H}_{3}\right)$ holds, then system $\left.(34)\right|_{\delta=0}$ becomes

$$
\begin{gathered}
\frac{d u}{d \tau}=-v+2 a_{1} u v+a_{1} u^{2} v+a_{3} v^{3} \\
\frac{d v}{d \tau}=u+\frac{3}{2} u^{2}+2 a_{4} u v+a_{5} v^{2}+\frac{1}{2} u^{3}+a_{4} u^{2} v \\
+a_{5} u v^{2}+a_{6} v^{3}
\end{gathered}
$$

in which the coefficients satisfy condition $\left(\mathrm{H}_{3}\right)$.

For convenience, we let $a_{4}=b_{4} r$; then condition $\left(H_{3}\right)$ becomes

$$
\begin{gathered}
a_{1}=\frac{1}{2}\left(b_{4}^{2}+2 b_{4}^{2} r-2\right), \\
a_{3}=-\frac{1}{6} b_{4}\left(3 b_{4}^{3}-12 b_{4}-14 b_{4} r+8 b_{4}^{3} r-4 b_{4} r^{2}+4 b_{4}^{3} r^{2}\right), \\
a_{5}=\frac{1}{2}\left(2-b_{4}^{2}+2 r-2 b_{4}^{2} r\right), \\
a_{6}=\frac{1}{3}\left(4 b_{4} r-b_{4}^{3} r+2 b_{4} r^{2}-2 b_{4}^{3} r^{2}\right) .
\end{gathered}
$$

System (80) complex concomitant system belongs to the form of (38). Hence we can use formulas (28) and (29) of Theorem $\mathrm{A}$ and $\tau_{k}=p_{k}+q_{k}=p_{k}^{\prime}+q_{k}^{\prime}$ to compute periodic constants of system (80).

Theorem 22. If condition (81) holds (i.e., $\left(\mathrm{H}_{3}\right)$ holds), then the first three period constants of the origin of system (80) are as follows:

$$
\tau_{1}=\frac{1}{6} l_{1}, \quad \tau_{2}=\frac{1}{96} l_{2}, \quad \tau_{3}=\frac{1}{414720} l_{3},
$$

in which $l_{i}, i=1,2,3,4$ are the polynomials of $b_{4}, r$. These expressions of $l_{i}$ are as follows:

$$
\begin{aligned}
l_{1}= & -24+3 b_{4}^{2}-15 r+18 b_{4}^{2} r-3 b_{4}^{4} r-2 r^{2} \\
& +4 b_{4}^{2} r^{2}-6 b_{4}^{4} r^{2}
\end{aligned}
$$

$$
\begin{aligned}
& l_{2}=336-234 b_{4}^{2}+63 b_{4}^{4}-6 b_{4}^{6}+1650 r-1284 b_{4}^{2} r \\
& +486 b_{4}^{4} r-90 b_{4}^{6} r+6 b_{4}^{8} r+1565 r^{2}-2370 b_{4}^{2} r^{2} \\
& +1124 b_{4}^{4} r^{2}-234 b_{4}^{6} r^{2}+19 b_{4}^{8} r^{2}+516 r^{3} \\
& -1224 b_{4}^{2} r^{3}+1184 b_{4}^{4} r^{3}-224 b_{4}^{6} r^{3}+4 b_{4}^{8} r^{3} \\
& +52 r^{4}-168 b_{4}^{2} r^{4}+320 b_{4}^{4} r^{4}-136 b_{4}^{6} r^{4}-20 b_{4}^{8} r^{4} \text {; } \\
& l_{3}=-12414600+10901385 b_{4}^{2}-4216185 b_{4}^{4} \\
& +963495 b_{4}^{6}-131355 b_{4}^{8}+8100 b_{4}^{10} \\
& -43826805 r+68754285 b_{4}^{2} r-37919070 b_{4}^{4} r \\
& +11032200 b_{4}^{6} r-1939005 b_{4}^{8} r+195615 b_{4}^{10} r \\
& -8100 b_{4}^{12} r-80932512 r^{2}+169994130 b_{4}^{2} r^{2} \\
& -121184220 b_{4}^{4} r^{2}+41520240 b_{4}^{6} r^{2}-7909800 b_{4}^{8} r^{2} \\
& +903798 b_{4}^{10} r^{2}-53460 b_{4}^{12} r^{2}-78594084 r^{3} \\
& +200907300 b_{4}^{2} r^{3}-192468840 b_{4}^{4} r^{3}+78954480 b_{4}^{6} r^{3} \\
& -15231180 b_{4}^{8} r^{3}+1650156 b_{4}^{10} r^{3}-138600 b_{4}^{12} r^{3} \\
& -38815064 r^{4}+119144360 b_{4}^{2} r^{4}-150195520 b_{4}^{4} r^{4} \\
& +82363760 b_{4}^{6} r^{4}-17141720 b_{4}^{8} r^{4}+1412136 b_{4}^{10} r^{4} \\
& -196560 b_{4}^{12} r^{4}-9033312 r^{5}+33223520 b_{4}^{2} r^{5} \\
& -55560320 b_{4}^{4} r^{5}+40281280 b_{4}^{6} r^{5}-11183680 b_{4}^{8} r^{5} \\
& +674208 b_{4}^{10} r^{5}-185760 b_{4}^{12} r^{5}-754208 r^{6} \\
& +3300480 b_{4}^{2} r^{6}-7720960 b_{4}^{4} r^{6}+7271040 b_{4}^{6} r^{6} \\
& -2734560 b_{4}^{8} r^{6}+181632 b_{4}^{10} r^{6}-97920 b_{4}^{12} r^{6} \text {. }
\end{aligned}
$$

Through analyzing the expressions of $\tau_{i}, i=1,2,3$ of Theorem 22, we have the following theorem.

Theorem 23. Note that $\tau_{1}=\tau_{2}=\tau_{3}=0$ of Theorem 22 have not solutions.

Proof. By computing carefully in personal computer, we obtain

$$
\begin{aligned}
R_{1}= & \text { Resultant }\left[l_{1}, l_{2}, r\right] \\
= & 9216\left(1+b_{4}^{2}\right)^{2}\left(4+5 b_{4}^{2}+8 b_{4}^{4}+b_{4}^{6}\right) \\
& \times\left(1-15 b_{4}^{2}-72 b_{4}^{4}+731 b_{4}^{6}+581 b_{4}^{8}\right. \\
& \left.-218 b_{4}^{10}-2 b_{4}^{12}+4 b_{4}^{14}\right),
\end{aligned}
$$




$$
\begin{aligned}
& R_{2}=\text { Resultant }\left[l_{1}, l_{3}, r\right]=-2654208\left(1+b_{4}^{2}\right)^{2} \\
& \times(-349081952+4306069852 b_{4}^{2} \\
&+41094839709 b_{4}^{4}-169659957471 b_{4}^{6} \\
&-996450752748 b_{4}^{8}-1647467327229 b_{4}^{10} \\
&-1097111490891 b_{4}^{12}+618270753348 b_{4}^{14} \\
&+1604753703528 b_{4}^{16}+406555036384 b_{4}^{18} \\
&-507767377067 b_{4}^{20}-144869567793 b_{4}^{22} \\
&+23948931114 b_{4}^{24}+2359097649 b_{4}^{26} \\
&\left.-735877143 b_{4}^{28}-4379940 b_{4}^{30}+7310250 b_{4}^{32}\right)
\end{aligned}
$$

in which Resultant $\left[l_{i}, l_{m}, r\right]$ is the resultant of $l_{i}$ and $l_{m}$ with respect to $r$.

Clearly, $R_{1}=R_{2}=0$ hold only if $1+b_{4}^{2}=0$; namely, $b_{4}= \pm i$. If $b_{4}= \pm i$, letting $\tau_{1}=0$, then $r=-3 / 2$. At this time, $a_{4}=b_{4} r= \pm(3 / 2) i$, which contradict $a_{4} \in \mathbf{R}$. Hence the conclusion of Theorem 23 holds. Proof ends.

From Theorem 23, equation groups $\tau_{1}=\tau_{2}=\tau_{3}=$ 0 have not solutions. Hence under condition $\left(H_{3}\right)$, the origin of system (80) cannot become an isochronous center. Accordingly, singular point $(-1 / 2,0)$ of system (2) cannot become an isochronous center under condition $\left(\mathrm{H}_{3}\right)$.

Remark 24. Through the above analysis, it is clear that the singular point $(-1 / 2,0)$ of system $(2)$ becomes an isochronous center if and only if $\left(H_{1}\right)$ and one of the conditions $\left(C_{1}\right)$ and $\left(C_{2}\right)$ hold.

\section{Conflict of Interests}

The author declares that there is no conflict of interests regarding the publication of this paper.

\section{Acknowledgments}

This research is partially supported by the National Natural Science Foundation of China (11371373 and 11261013) and the Research Fund of Hunan Provincial Education Department and Hunan provincial Science and Technology Project (2012FJ3106).

\section{References}

[1] Y. Lin and J. Li, "Normal form and critical points values of the period of closed orbits for planar autonomous systems," Acta Mathematica Sinica, vol. 34, no. 4, pp. 490-501, 1991.

[2] V. G. Romanovskii, "Calculation of Lyapunov numbers in the case of two pure imaginary roots," Differential Equations, vol. 29, no. 5, pp. 782-784, 1993.

[3] W. Huang, Y. Liu, and W. Zhang, "Conditions of infinity to be an isochronous centre for a rational differential system,"
Mathematical and Computer Modelling, vol. 46, no. 5-6, pp. 583594, 2007.

[4] Y. Liu and W. Huang, "Center and isochronous center at infinity for differential systems," Bulletin des Sciences Mathematiques, vol. 128, no. 2, pp. 77-89, 2004.

[5] L. Feng and L. Yirong, "Classification of the centers and isochronicity for a class of quartic polynomial differential systems," Communications in Nonlinear Science and Numerical Simulation, vol. 17, no. 6, pp. 2270-2291, 2012.

[6] F. Li, J. Qiu, and J. Li, "Bifurcation of limit cycles, classification of centers and isochronicity for a class of non-analytic quintic systems," Nonlinear Dynamics, vol. 76, no. 1, pp. 183-197, 2014.

[7] V. G. Romanovski and D. S. Shafer, The Center and Cyclicity Problems. A Computational Algebra Approach, Birkhäuser, Berlin, Germany, 2009.

[8] V. G. Romanovski and M. Robnik, "The centre and isochronicity problems for some cubic systems," Journal of Physics A: Mathematical and General, vol. 34, no. 47, pp. 10267-10292, 2001.

[9] V. G. Romanovski, X. Chen, and Z. Hu, "Linearizability of linear systems perturbed by fifth degree homogeneous polynomials," Journal of Physics A: Mathematical and Theoretical, vol. 40, no. 22, pp. 5905-5919, 2007.

[10] J. Giné and V. G. Romanovski, "Integrability conditions for Lotka-Volterra planar complex quintic systems," Nonlinear Analysis: Real World Applications, vol. 11, no. 3, pp. 2100-2105, 2010.

[11] X. Chen and V. G. Romanovski, "Linearizability conditions of time-reversible cubic systems," Journal of Mathematical Analysis and Applications, vol. 362, no. 2, pp. 438-449, 2010.

[12] L. Cairó, J. Chavarriga, J. Giné, and J. Llibre, "Class of reversible cubic systems with an isochronous center," Computers and Mathematics with Applications, vol. 38, no. 11, pp. 39-53, 1999.

[13] A. Gasull, A. Guillamon, and V. Mañosa, "An explicit expression of the first Liapunov and period constants with applications," Journal of Mathematical Analysis and Applications, vol. 211, no. 1, pp. 190-212, 1997.

[14] W. S. Loud, "Behavior of the period of soutions of certain plane autonomous systems near centers," Contributions to Differential Equations, vol. 3, pp. 21-36, 1964.

[15] I. Pleshkan, "A new method of investigating the isochronicity of system of two differential equations," Differential Equations, vol. 5, pp. 796-802, 1969.

[16] C. Du, Y. Liu, and H. Mi, "A class of ninth degree system with four isochronous centers," Computers and Mathematics with Applications, vol. 56, no. 10, pp. 2609-2620, 2008.

[17] C. X. Du, H. L. Mi, and Y. R. Liu, "Center, limit cycles and isochronous center of a Z4-equivariant quintic system," Acta Mathematica Sinica, vol. 26, no. 6, pp. 1183-1196, 2010.

[18] F. Li and M. Wang, "Bifurcation of limit cycles in a quintic system with ten parameters," Nonlinear Dynamics, vol. 71, pp. 213-222, 2013.

[19] Y. Liu and J. Li, "Periodic constants and time-angle difference of isochronous centers for complex analytic systems," International Journal of Bifurcation and Chaos, vol. 16, no. 12, pp. 37473757, 2006.

[20] Y. Liu, "Theory of center-focus for a class of higher-degree critical points and infinite points," Science in China A: Mathematics, Physics, Astronomy, vol. 44, no. 3, pp. 365-377, 2001.

[21] V. V. Amel'kin, N. A. Lukashevich, and A. P. Sadovskii, Nonlinear Oscillations in Second Order Systems, Belarusian State University, Minsk, Belarus, 1982. 


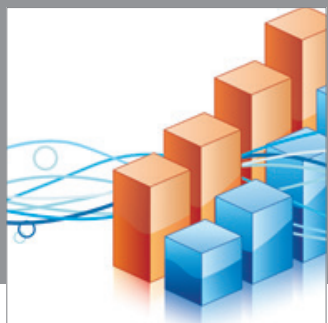

Advances in

Operations Research

mansans

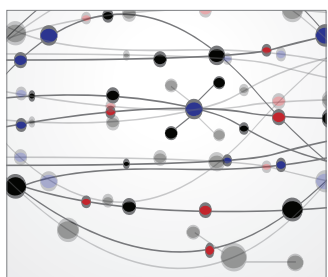

The Scientific World Journal
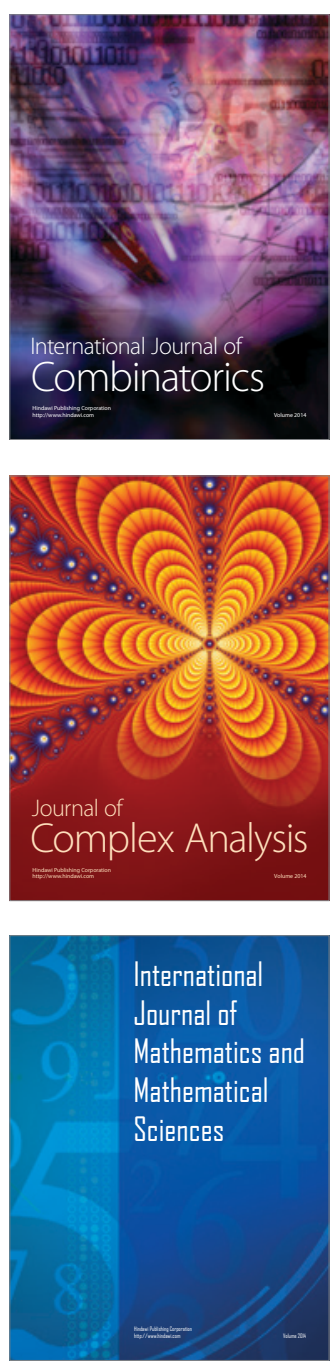
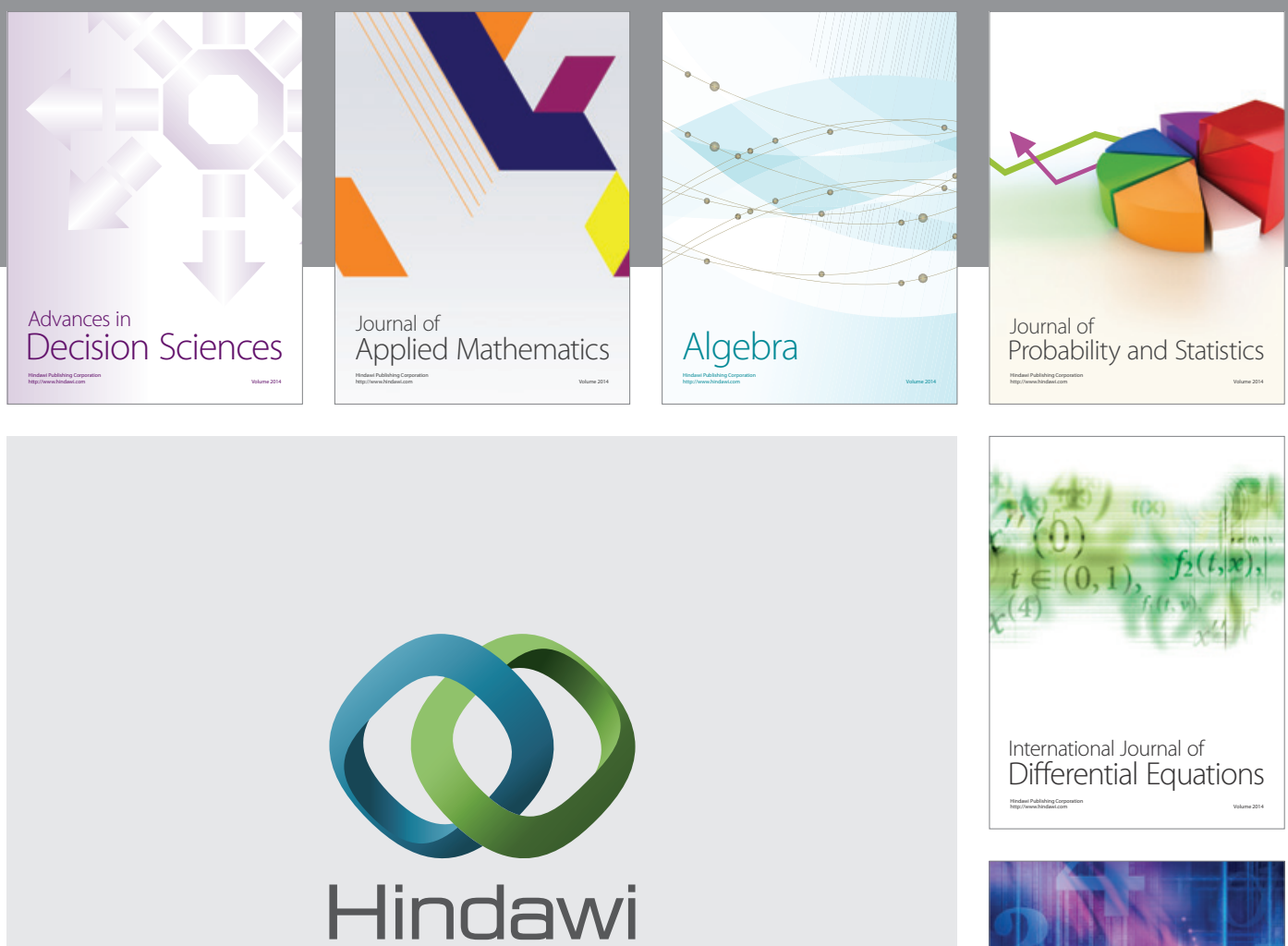

Submit your manuscripts at http://www.hindawi.com
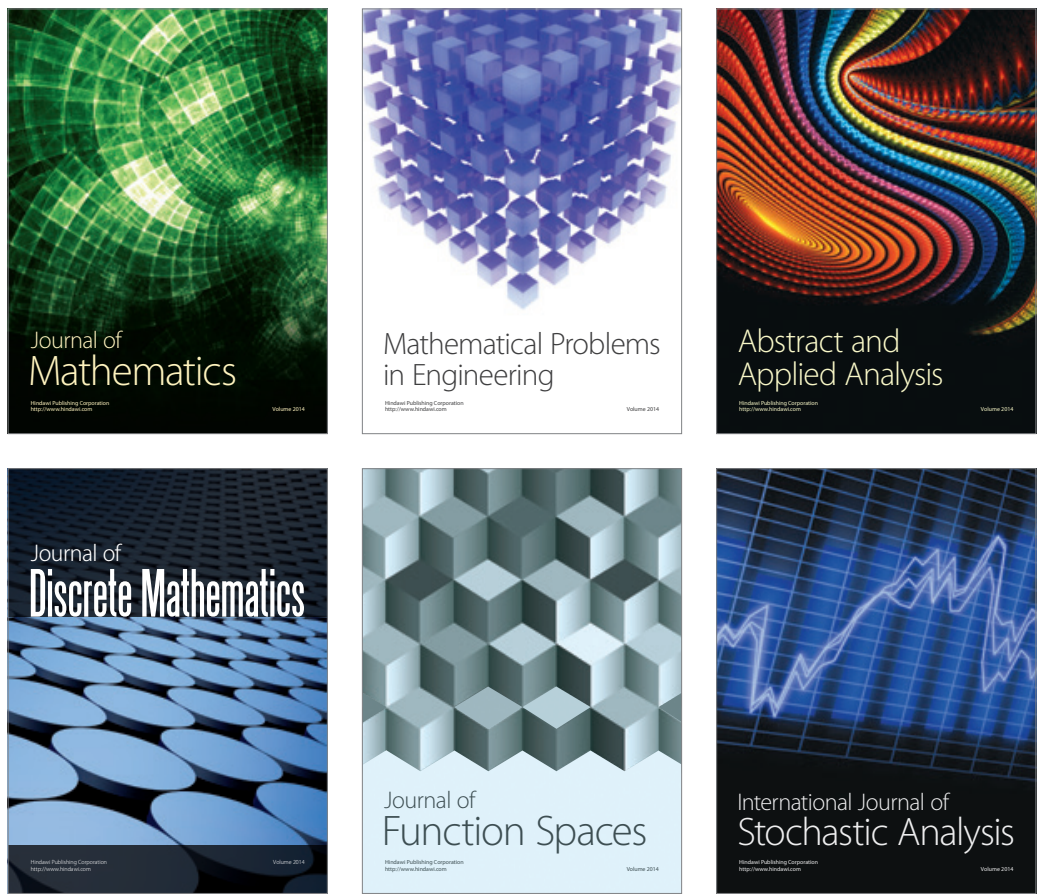

Journal of

Function Spaces

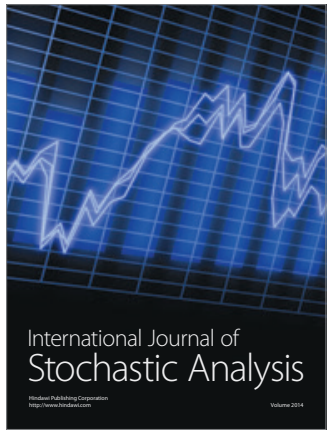

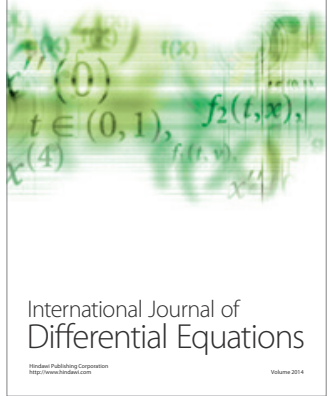
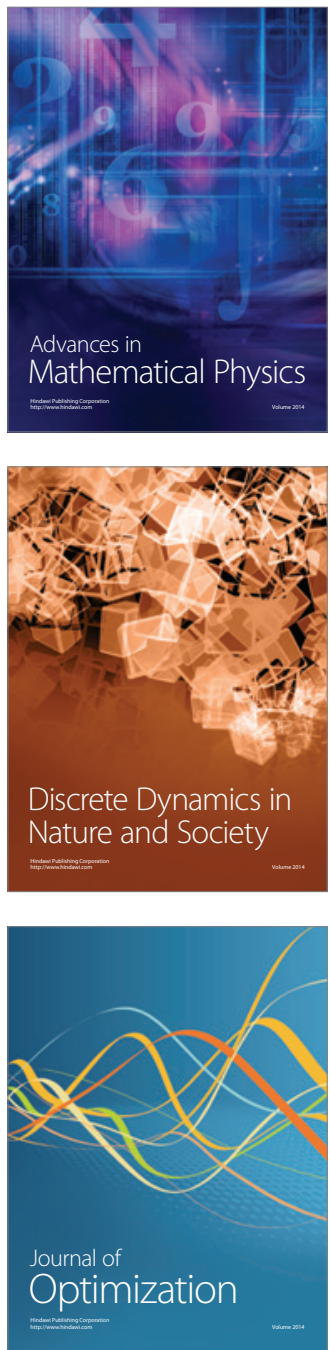